\title{
FINITE-TEMPERATURE LATTICE QCD WITH WILSON FERMIONS
}

\author{
T. ÇELIK', J. ENGELS and H. SATZ \\ Fakultät für Physik, Universität Bielefeld, D-4800 Bielefeld, West Germany
}

Received 20 November 1984

\begin{abstract}
The thermodynamics of QCD with dynamical Wilson fermions is studied in a low-order hopping parameter expansion, using Monte Carlo simulation on $8^{3} \times 3$ to $10^{3} \times 5$ lattices. We observe a clear deconfinement transition at $T_{\mathrm{c}} / A_{\mathrm{L}}^{N_{r}-2} \simeq 150$; chiral symmetry restoration occurs at the same point. Within our approximation, both transitions are continuous. In the confinement regime, we find the global centre $Z_{3}$ symmetry only very weakly broken, in accord with a picture relating string breaking in QCD with ionization in insulating solids.
\end{abstract}

\section{Introduction}

The thermodynamics of pure $\mathrm{SU}(N)$ gauge fields predicts the occurrence of a deconfining phase transition [1]. At some temperature $T_{\mathrm{c}}$, colour screening dissolves the confining bond and turns gluonium matter into a chromoplasma [2]. One of the most interesting problems in current statistical QCD is what effect the introduction of dynamical quarks has on this deconfinement.

On a phenomenological level, colour screening considerations appear to remain valid; the presence of many other colour charges should dissolve bound quark states just as well as gluonium states. On the other hand, the global symmetry under the centre $Z_{N}$ of the $S U(N)$ gauge group is broken by the fermion term in the lagrangian, and hence deconfinement can no longer be strictly characterized in terms of spontaneous $Z_{N}$ symmetry breaking. The expectation value $\bar{L}$ of the thermal Wilson loop does not any more constitute an order parameter for the deconfinement transition, since it now does not vanish in the confinement region. However, it is unclear how much $\bar{L}$ differs from zero there, i.e. how strongly the $Z_{N}$ symmetry is actually broken. We must therefore ask by what mechanism $\bar{L}$ is determined in the confinement zone.

The deconfinement transition is the chromodynamic analog of the metal-insulator transition [3] in systems with electromagnetic forces. In insulating solids, the electric conductivity $\sigma$ is not strictly zero, but only exponentially small [3]:

$$
\sigma \sim \exp \left\{-E_{i} / T\right\},
$$

where $E_{i}$ denotes the ionization energy. Above the transition point to a metal, $\sigma$ is non-zero because Debye screening has globally dissolved the Coulomb binding

A.v. Humboldt Fellow, now at Hacettepe University, Ankara, Turkey. 
between ions and electrons; but even below this point, ionization can locally provide some free electrons, and thus make $\sigma>0$. The corresponding phenomenon in QCD with dynamical quarks is the production of qã pairs. In pure gauge theory below the deconfinement temperature, an infinite energy is needed to break up a static quark-antiquark pair; this corresponds to an infinite ionization energy in eq. (1). With dynamic quarks, such a break-up becomes possible when the separation yields a binding energy equal to that needed to produce a $q \bar{q}$ pair, i.e. a hadron. The newly-formed quarks neutralize the static quarks and thus allow their separation. We therefore expect that $\bar{L}$ will now no longer vanish in the confinement zone, but that

$$
\bar{L} \sim \exp \left\{-m_{\mathrm{H}} / 2 T\right\},
$$

where $m_{H}$ is the mass of the dominant qā bound state. Letting $m_{H} \rightarrow \infty$ in eq. (2), we recover the pure gauge result.

Both the conventional Mott transition in solids and the deconfinement transition in hadronic matter thus lead from a regime, in which the binding can locally be broken (by ionization or $\mathrm{q} \overline{\mathrm{q}}$ formation, respectively) to one, where it is globally removed by a collective screening of the binding force. Although this provides us with an intuitive picture of how an $\bar{L} \neq 0$ arises in the confinement region, it does not allow us to estimate it quantitatively. Thus also the question of the sharpness of the transition, or of its order, if it still is a genuine phase transition, remains open.

For colour SU(3), the deconfinement transition of the pure gauge theory is of first order [4]. Hence very heavy quarks will not totally remove the discontinuities in thermodynamic observables. Massive constituents are thermodynamically suppressed, and only if the quark mass becomes small enough can the deconfinement pattern of the gluon system be changed.

However, quark mass considerations around the deconfinement point remain rather arbitrary as long as the relation between chiral symmetry restoration and deconfinement is not clarified. In a state of broken chiral symmetry, the effective quark mass is not zero, and if it is large enough, we may still have a first-order deconfinement transition [5].

On the other hand, the role of the quark term in the QCD lagrangian is similar to that of an external magnetic field applied to a spin system [6-9]. This immediately shows why $\bar{L}$ should cease to vanish in the confinement zone, although it does not directly relate its value there with any physical quantity. The discontinuity in $\bar{L}$ decreases with decreasing quark mass (increasing external field), and based on strong coupling arguments it has been suggested that the deconfinement transition may be completely washed out in the light-quark limit [6-9]. However, all quantitative studies performed so far [10-13] continue to show a very abrupt deconfinement transition even for the lightest quark masses considered.

To study this problem in more detail, we have performed numerical calculations with Wilson fermions [14] of two flavours in a low-order hopping parameter 
expansion, on lattices ranging from $8^{3} \times 3$ to $10^{3} \times 5$. We have chosen this fermion formulation because it allows in reasonable time calculations with rather good statistics on fairly large lattices. Moreover, the Wilson fermion scheme even in the lowest-order hopping parameter expansion contains all the essential features used to argue for the disappearance of the deconfinement transition. Therefore, if we understand here why it persists to such an extent, then this will be applicable to other fermion formulations as well; and as we have already noted, so far the different fermion schemes do lead to very similar results on deconfinement and chiral symmetry restoration.

In the next section, we shall sketch the formalism of statistical QCD on the lattice, using the Wilson fermion scheme. In sect. 3 we then present our numerical results for the relevant thermodynamic observables.

\section{Statistical QCD with Wilson fermions}

Our starting point is the euclidean lattice form of the QCD partition function:

$$
Z_{\mathrm{F}}(\beta)=\int \prod_{\text {links }} \mathrm{d} U\{\operatorname{det}(1-\kappa M)\}^{N_{f}} \exp \left[-S_{\mathrm{G}}(U)\right],
$$

as it appears after integration of the quark spinor fields. Here

$$
S_{\mathrm{G}}(U)=\left\{\frac{6}{g_{\beta}^{2}}\left(\frac{a_{\sigma \sigma}}{a_{\beta}}\right) \sum_{\mathrm{P}_{\beta}}\left(1-\frac{1}{3} \operatorname{Re} \operatorname{tr}(U U U U)\right)+\frac{6}{g_{\sigma}^{2}}\left(\frac{a_{\beta}}{a_{\sigma}}\right) \sum_{\mathrm{P}_{\sigma}}\left(1-\frac{1}{3} \operatorname{Re} \operatorname{tr}(U U U U)\right)\right\}
$$

is the Wilson action for $\mathrm{SU}(3)$ gauge fields at finite temperature [15], obtained by summing over space-time $\left(\mathrm{P}_{\beta}\right)$ and space-space plaquettes $\left(\mathrm{P}_{\sigma}\right) ; a_{\sigma}$ and $a_{\beta}$ are the spatial and temporal lattice spacings, $g_{c}$ and $g_{\beta}$ the corresponding couplings. The form (3) holds for $N_{f}$ flavours of quarks with equal mass. The matrix $M$

$$
M_{\mu, n m}=\left(1-\gamma_{\mu}\right) U_{n m} \delta_{n, m-\hat{\mu}}+\left(1+\gamma_{\mu}\right) U_{m n}^{+} \delta_{n, m+\hat{\mu}}
$$

describes the interaction for each flavour, corresponding to the Wilson form of the fermion action

$$
S_{\mathrm{F}}(U)=\sum_{f} \bar{\psi}_{f}(1-\kappa M) \psi_{f}
$$

in eq. (5), $\hat{\mu}$ is a unit vector along a lattice link. In the finite temperature case, the hopping parameter $\kappa\left(g^{2}\right)$ depends on the link direction

$$
\kappa M \equiv \kappa_{\beta} M_{0}+\kappa_{\sigma} \sum_{\mu=1}^{3} M_{\mu} ;
$$

however, for $a_{\sigma}=a_{\beta}=a, \kappa_{\beta}=\kappa_{\sigma}=\kappa\left(g^{2}\right)$. 
We now expand the logarithm of $\operatorname{det}(1-\kappa M)$ in powers of the hopping parameter [16]

$$
\ln \operatorname{det}(1-\kappa M)=-\operatorname{Tr} \sum_{l=1}^{\infty} \frac{\kappa^{\prime}}{l} M^{l} .
$$

Only closed loops contribute to eq. (8). The inclusion of the fermion determinant thus corresponds to having in eq. (3) an effective action

$$
S_{\text {eff }}(U)=S_{G}(U)-4 N_{f}(2 \kappa)^{N_{s}} \sum_{\text {sites }} \operatorname{Re} L-16 N_{f} \kappa^{4} \sum_{\mathrm{P}_{\theta}, \mathrm{P}_{t r}} \operatorname{Re} \operatorname{Tr}(U U U U)+\mathrm{O}\left(\kappa^{5}\right) .
$$

Since the third term on the r.h.s of eq. (9) simply shifts the coupling by

$$
\frac{6}{g^{2}} \rightarrow \frac{6}{g^{2}}+48 N_{f} \kappa^{4}
$$

the main effect of the presence of fermions is contained in the thermal Wilson loop term of $S_{\text {eff }}$, with

$$
L(x) \equiv \operatorname{Tr}\left\{\prod_{\tau=1}^{N_{B}} U_{x ; \tau, \tau+1}\right\} .
$$

The hopping parameter $\kappa\left(g^{2}\right)$ is for massless quarks given at small $g^{2}$ by [17]

$$
\kappa\left(g^{2}\right)=\frac{1}{8}\left[1+0.11 g^{2}+O\left(g^{4}\right)\right] .
$$

At the $g^{2}$ value we have considered, we expect $10-20 \%$ deviations from the weak coupling behaviour (12); in fig. 1, we show Monte Carlo results obtained by requiring $m_{\pi}=0$, together with eq. (12) up to order $g^{2}$. Since the form (12) falls below the critical Monte Carlo curve, it corresponds to quarks of non-vanishing mass. This keeps us within the radius of convergence of the hopping parameter expansion. For similar reasons, other fermion schemes also use finite quark masses in the actual calculation. We have thus used the form (12), as we also use the renormalization group relation

$$
a \Lambda_{\mathrm{L}}=\exp \left\{-\frac{4 \pi^{2}}{33-2 N_{f}}\left(\frac{6}{g^{2}}\right)+\frac{459-57 N_{f}}{\left(33-2 N_{f}\right)^{2}} \log \left[\frac{8 \pi^{2}}{33-2 N_{f}}\left(\frac{6}{g^{2}}\right)\right]\right\},
$$

obtained from the weak coupling expansion of the $\beta$-function,

$$
-\beta\left(g^{2}\right)=\frac{\left(33-2 N_{f}\right)}{3(4 \pi)^{2}} g^{3}\left[1+\frac{\left(306-38 N_{f}\right)}{(4 \pi)^{2}\left(33-2 N_{f}\right)} g^{2}+O\left(g^{4}\right)\right] .
$$

It appears reasonable to expect both eq. (12) and eq. (14) to apply well for $g^{2} \leqslant 1$ (see for example [18] for a survey of recent results of scaling tests).

From the partition function (3) we now get the energy density $\varepsilon$ as a function of the temperature. With $\varepsilon \equiv \varepsilon_{\mathrm{G}}+\varepsilon_{\mathrm{F}}$, we have for $a_{o}=a_{\beta}$

$$
\varepsilon_{\mathrm{G}} / T^{4}=18 N_{\beta}^{4}\left\{g^{-2}\left(\overline{\mathrm{P}}_{\sigma}-\overline{\mathrm{P}}_{\beta}\right)+c_{\sigma}^{\prime}\left(\overline{\mathrm{P}}-\overline{\mathrm{P}}_{\sigma}\right)+c_{\beta}^{\prime}\left(\overline{\mathrm{P}}-\overline{\mathrm{P}}_{\beta}\right)\right\}
$$




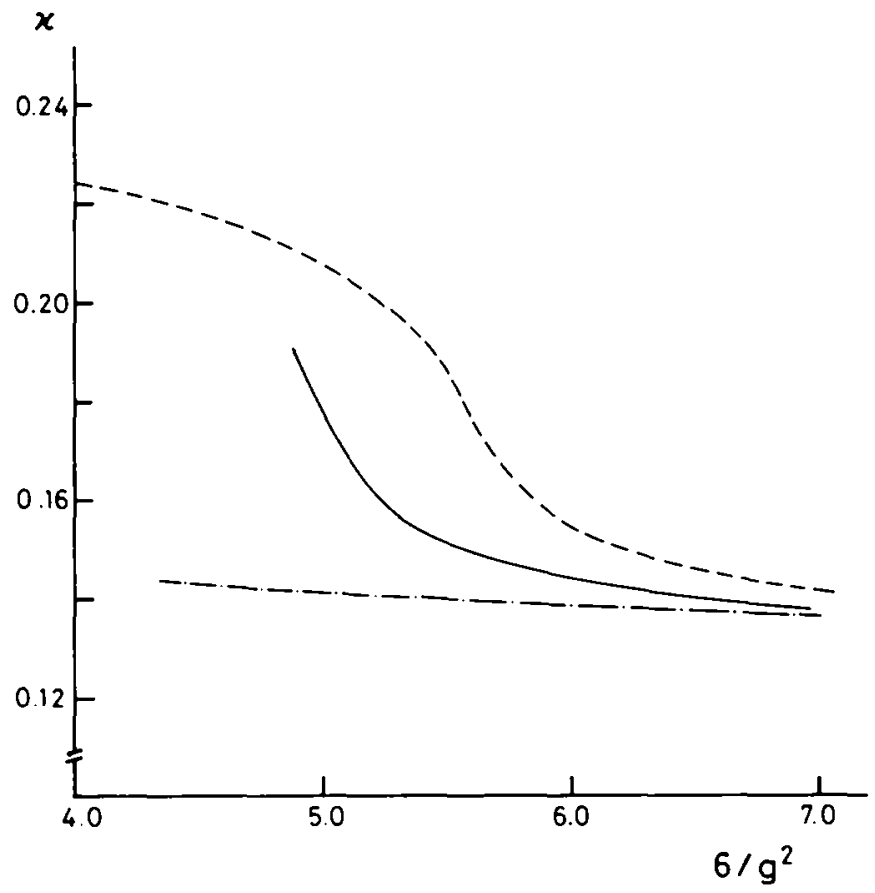

Fig. 1. Hopping parameter $\kappa$ as function of $6 / \mathrm{g}^{2}$, for the quenched case (the dashed curve, obtained by interpolation of numerical data), for the case $N_{f}=2$ (the solid curve, obtained from the previous curve by use of the renormalization group relation), and the weak coupling form (dash-dotted curve, obtained from relation (12)).

for the gluon sector and

$$
\varepsilon_{\mathrm{F}} / T^{4}=N_{\beta}^{4} N_{f}\left\{3(2 \kappa)^{N_{\beta}} \operatorname{Re} \bar{L}+144 \kappa^{4}\left(\overline{\mathrm{P}}_{\sigma}-\overline{\mathrm{P}}_{\beta}\right)+\mathrm{O}\left(\kappa^{5}\right)\right\}
$$

for the fermion sector. The separation of $\varepsilon$ into two such terms corresponds to the two components of $S_{\text {eff }}$; in the interaction regime, an actual separation into gluon and quark systems is of course impossible. In eq. (15), $\overline{\mathrm{P}}_{\sigma}$ and $\overline{\mathrm{P}}_{\beta}$ denote the space-space and space-temperature plaquette averages on the $N_{\sigma}^{3} \times N_{\beta}$ lattice; $\overline{\mathrm{P}}$ is the average on a large $\left(N_{\sigma}^{4}\right)$ symmetric lattice. The constants $c_{\sigma}^{\prime}$ and $c_{\beta}^{\prime}$ come from differentiating the couplings $g_{\sigma}$ and $g_{\beta}$ with respect to $a_{\beta}$; for colour SU(3) and Wilson fermions of two flavours, one has [19] $c_{\sigma}^{\prime}=0.19366$ and $c_{\beta}^{\prime}=-0.132463$. In eq. (16), $\bar{L}$ denotes the lattice average of the thermal Wilson loop.

The energy density thus determined will certainly display finite-size effects - both because of the lattice regularization [20] and because of the truncation of the hopping parameter expansion. To compare our results with those of a non-interacting quarkgluon system, we therefore calculate the ideal gas forms $\varepsilon_{\mathrm{G}}^{\mathrm{SB}}$ and $\varepsilon_{r}^{\mathrm{SB}}$ on lattices of the same size and in the same order of hopping parameter expansion. For $\varepsilon_{\mathrm{G}}$, the 
resulting values are given in ref. [20]; for $\varepsilon_{\mathrm{F}}^{\mathrm{SB}}$ we have from eq. (16) by setting all $U=1$

$$
\varepsilon_{\mathrm{F}}^{\mathrm{SB}} / T^{4}=9 N_{\beta}^{4} N_{f}(4)^{-N_{\beta}},
$$

where we have taken $\kappa^{\mathrm{SB}}=\frac{1}{8}$ and neglected fifth and higher order contributions.

If the quark-gluon system undergoes a sudden transition from the hadronic to the plasma phase, we expect this to reflect strongly in the behaviour of $\varepsilon$. To study the restoration of chiral symmetry, we consider the order parameter [16]

$$
\begin{aligned}
\left.\langle\bar{\psi} \psi\rangle a^{3}\right]_{\text {per flavour }}= & 2 \kappa \sum_{l=0}^{\infty} \kappa^{\prime} \overline{\operatorname{Tr} M^{\prime}}=2 \kappa\left\{12-4(2 \kappa)^{N_{\beta}} \operatorname{Re} \bar{L}\right. \\
& \left.-1152 \kappa^{4}\left(1-\frac{1}{2}\left(\overline{\mathrm{P}}_{\sigma}+\overline{\mathrm{P}}_{\beta}\right)\right)+\mathrm{O}\left(\kappa^{5}\right)\right\} .
\end{aligned}
$$

Since in the Wilson formulation $\langle\bar{\psi} \psi\rangle$ never vanishes on a finite lattice, the relevant quantity to consider as chiral symmetry order parameter is

$$
\Delta\langle\bar{\psi} \psi\rangle \equiv\langle\bar{\psi} \psi\rangle-\langle\bar{\psi} \psi\rangle_{\mathrm{wC}},
$$

where in $\langle\bar{\psi} \psi\rangle_{\text {wC }}$ the weak coupling limits* for $\operatorname{Re} \bar{L}$ and the plaquette averages of ref. [21] are used. Chiral symmetry restoration is a non-perturbative effect, and by subtracting the perturbative form with our $\kappa$ (i.e. for finite mass), we can obtain an idea of where the restoration occurs.

The aim of our study will now be the numerical determination of the energy density $(15) /(16)$, the average of the thermal Wilson loop (11), and the chiral symmetry measure (19).

\section{Numerical results}

The major part of our results is obtained on an $8^{3} \times 3$ lattice, with about 2000 lattice sweeps (iterations) per value of the coupling; of these, the first 500 are generally discarded.

In fig. 2 we show the behaviour of the gluon sector energy density (15) as a function of $6 / g^{2}$, together with the Stefan-Boltzmann result on an $8^{3} \times 3$ lattice [20]. Also shown is the corresponding result for the pure gauge theory [22]. We note that the transition now occurs at a lower value of $6 / \mathrm{g}^{2}$; this is to be expected because of the modified renormalization group relation (13), if $T_{\mathrm{c}}$ is not significantly changed. In contrast to the pure gauge case, the transition now also has become continuous. Finally we note a rather sizeable overshoot in the transition region. This is, as we shall see more clearly in a moment, due to the $\operatorname{Re} \bar{L}$ part of the effective action (9); it causes a more complete alignment of the $U$ than would be the case in the pure gauge theory.

The energy density of the quark sector with $N_{f}=2$, shown in fig. 3, still falls short of the limit (17); the reason for this is that $\operatorname{Re} \bar{L}$ attains its asymptotic limit only

* The results of ref. [21] correspond to the quenched case; we do not expect significant changes in eq. (19) when the determinant is included. 


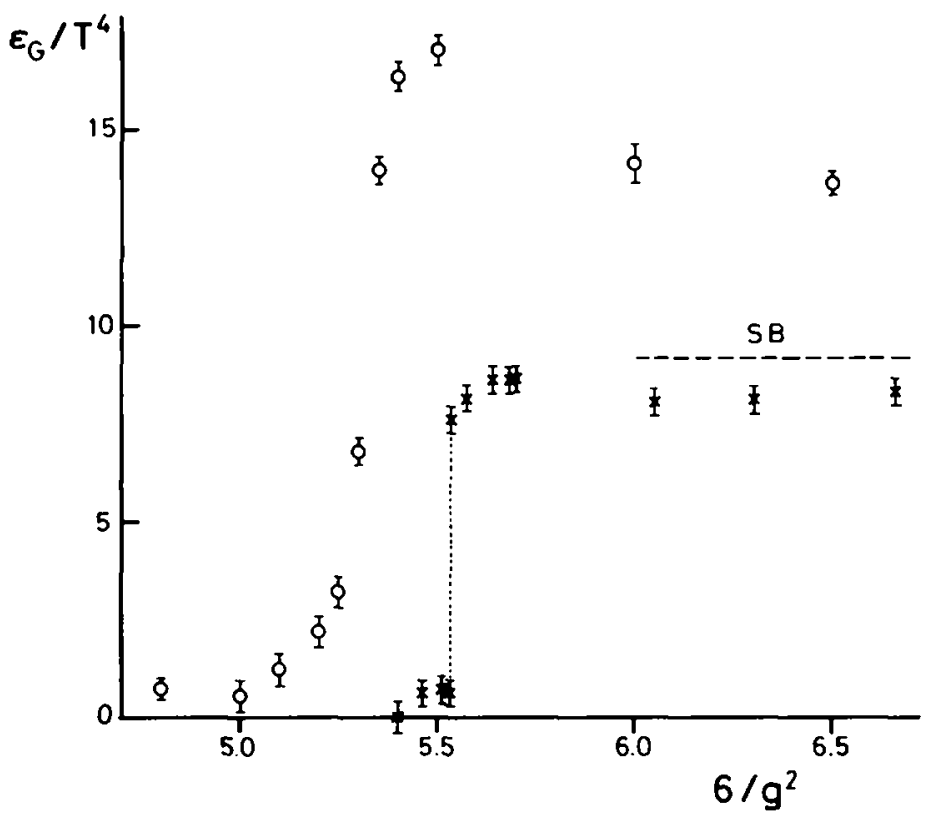

Fig. 2. The energy density $\varepsilon_{\mathrm{G}}$ for the gluon sector, calculated on an $8^{3} \times 3$ lattice in the fourth-order hopping parameter expansion (O). Also shown are the pure gauge theory result $(x)$ and the ideal gas limit (dashed line) on the same size lattice.

rather slowly, as is seen in fig. 4 . We might note at this point that within our error bars $\operatorname{Im} \bar{L}$ is essentially zero, even below the deconfinement point, where it is about $\frac{1}{10}$ or less of $\operatorname{Re} \bar{L}$.

Combining $\varepsilon_{\mathrm{G}}$ and $\varepsilon_{\mathrm{F}}$, we obtain the behaviour of the overall energy density. It is shown in fig. 5 as function of the temperature $T / \Lambda_{\mathrm{L}}$, obtained with the help of the renormalization group relation (13). The Stefan-Boltzmann limit in fig. 5 is the sum of the corresponding gluon and quark sector forms. It thus includes the effect both of finite lattice size and of fourth-order hopping parameter truncation. The latter only results in a $5 \%$ reduction of the complete series.

To obtain some feeling for the size of $\varepsilon$ in the confinement region, we also include in fig. 5 the energy density of an ideal gas of $\pi, \rho$ and $\omega$ mesons, using for illustration purposes $A_{L}^{N^{\prime}=2}=1.5 \mathrm{MeV}$ for the lattice scale in physical units. We see that such an ideal meson gas indeed leads to an $\varepsilon$ of the same order of magnitude as we obtain from our QCD evaluation.

We have already shown in fig. 4 the average thermal Wilson loop $\operatorname{Re} \bar{L}$; at the end of the deconfinement regime, it falls rather rapidly to a very small value. The behaviour of the corresponding fluctuation is given by the susceptibility

$$
\chi \equiv\left\{\overline{L^{2}}-\bar{L}^{2}\right\} N_{\sigma}^{3} ;
$$

and is shown in fig. 6 . The apparent strong increase at the critical point, together 


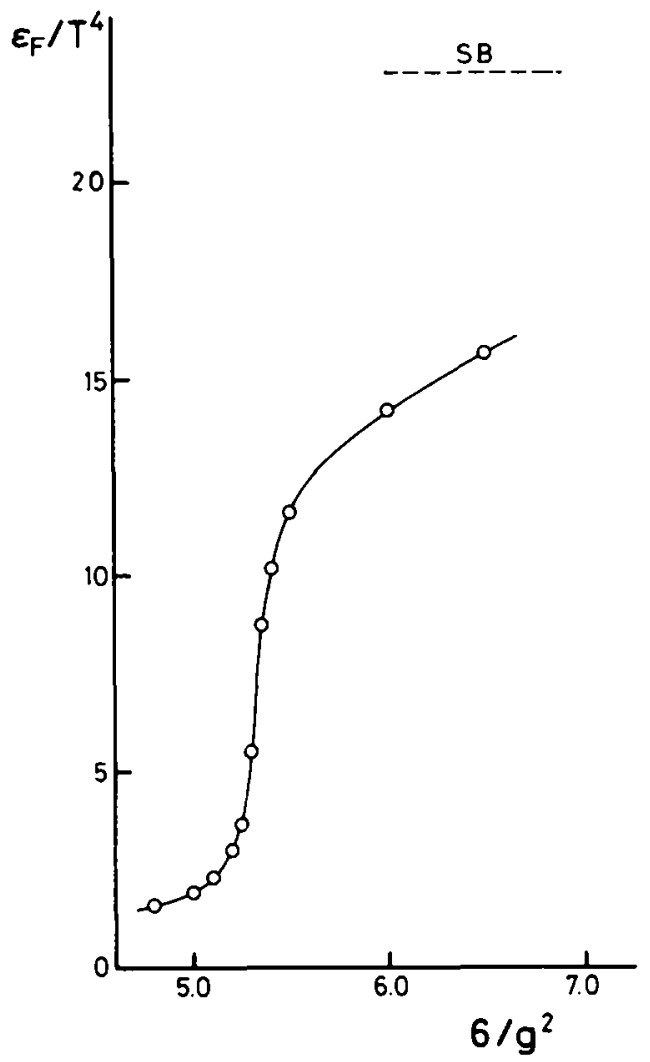

Fig. 3. The energy density $\varepsilon_{\mathrm{G}}$ for the fermion sector, calculated on an $8^{3} \times 3$ lattice in the fourth-order hopping parameter expansion $(O)$, together with the ideal gas limit on a lattice of the same size (dashed line).

with the rapid but smooth variation of both $\bar{L}$ and $\varepsilon$ suggests a second-order transition. Comparing the results of hot and cold starts around the deconfinement point, we have in fact not observed any two-state signal.

Finally we present in fig. 7 the behaviour of the chiral symmetry measure (19), together with that of $\bar{L}$. Deconfinement and chiral symmetry restoration are thus seen to occur at the same point.

Before we study the effect of higher orders and larger lattices, let us note here that the behaviour we have observed for Wilson fermions in fourth-order hopping parameter expansion agrees in all points with that observed for Kogut-Susskind fermions both in the microcanonical $[13]$ and in the canonical $[11,12]$ formulation.

The truncation of the hopping parameter expansion (8) at $l=4$ is clearly a rather drastic measure. To obtain at least some idea of the effect of higher-order terms, we have repeated our calculations including the next $\left(\kappa^{5}\right)$ order, again on an $8^{3} \times 3$ lattice. In fig. 8 we show the resulting change both in $\varepsilon$ and in $\bar{L}$; it does not seem 


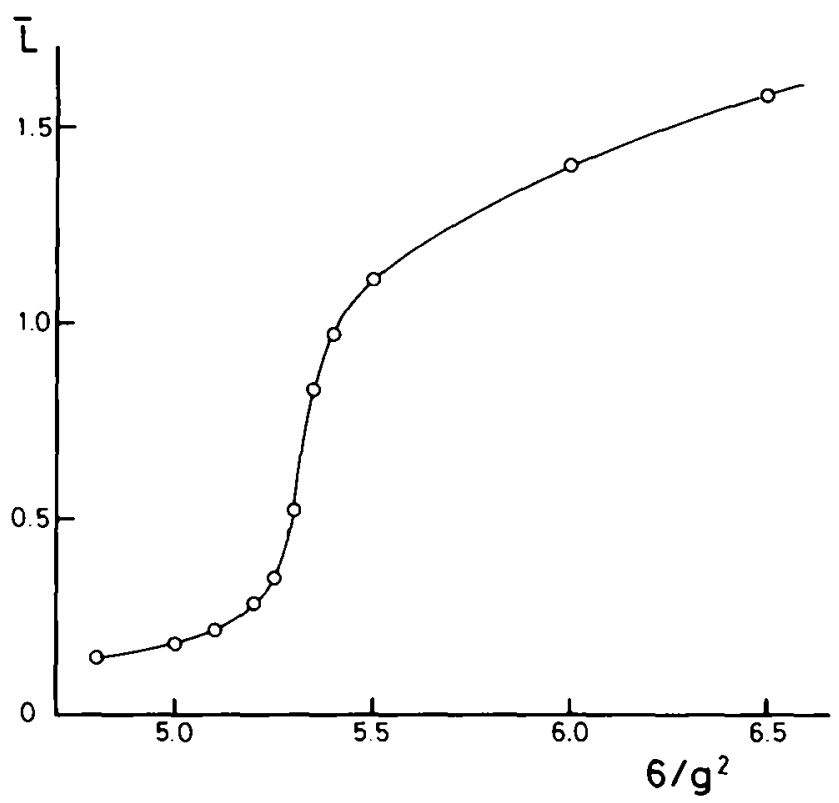

Fig. 4. The average of the thermal Wilson loop on an $8^{3} \times 3$ lattice as a function of $6 / \mathrm{g}^{2}$.

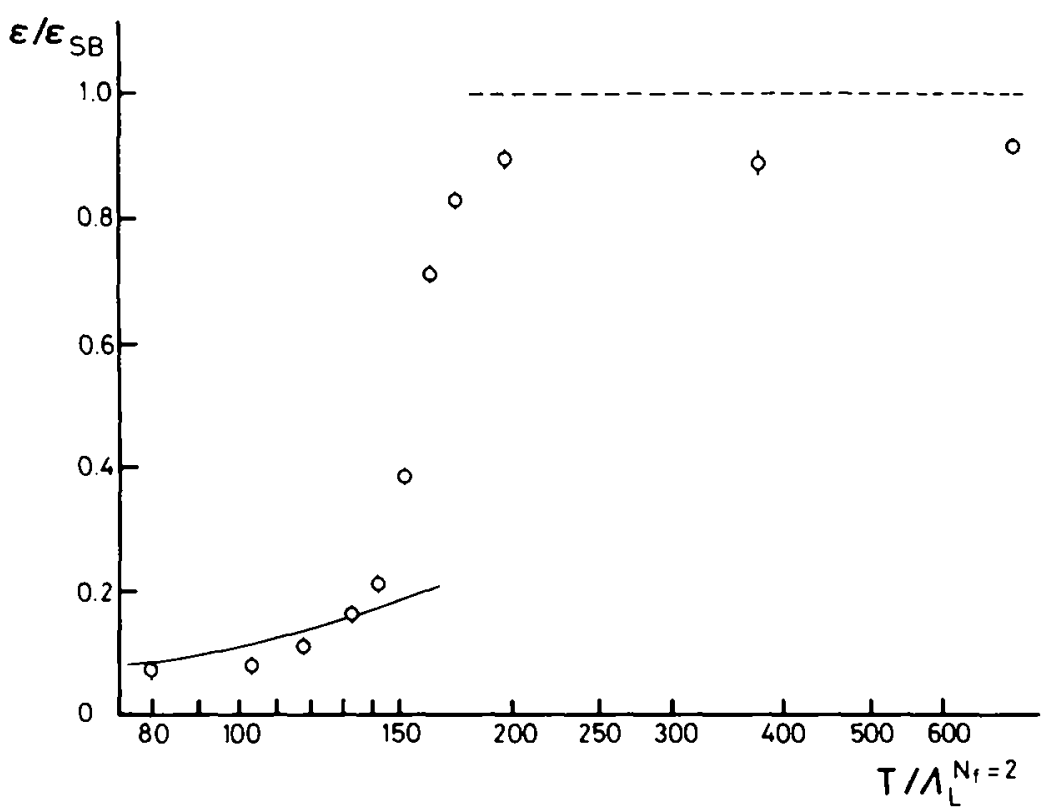

Fig. 5. The total energy density $\varepsilon$, normalized to the ideal gas limit $\varepsilon_{\mathrm{SB}}$, as a function of the temperature, calculated on an $8^{3} \times 3$ lattice in the fourth-order hopping parameter expansion. Also shown are the ideal gas limits for the quark-gluon plasma (dashed line) and for a system of $\pi, \rho$ and $\omega$ mesons (solid line). 


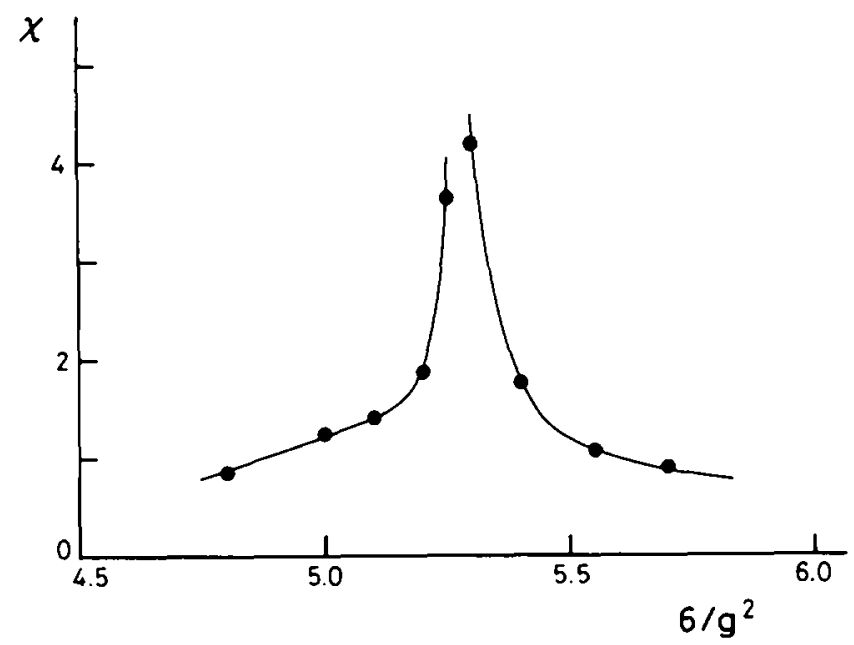

Fig. 6. The susceptibility $\chi$ in the fifth-order hopping parameter expansion, calculated on an $8^{3} \times 3$ lattice.

to effect our conclusions. We expect the role of higher-order terms to be most crucial in the determination of the actual value of the critical coupling; since the inclusion of higher orders results in a shift to smaller $6 / \mathrm{g}_{\mathrm{c}}^{2}$, our fourth-order results provide an upper bound for the critical temperature. We shall see shortly that this bound is not much above the corresponding $T_{\mathrm{c}}$ values in other fermion schemes [11-13].

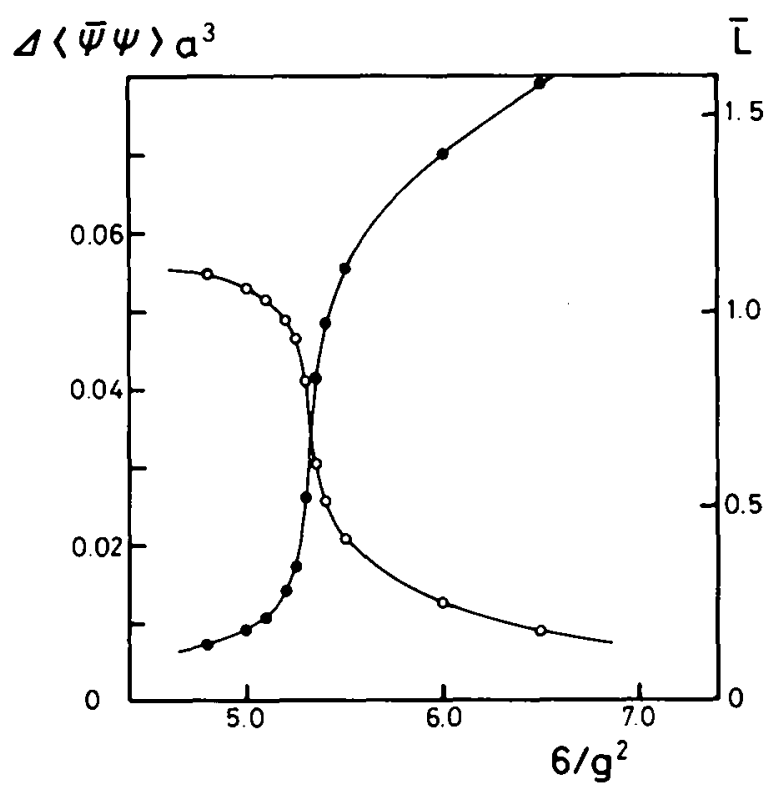

Fig. 7. Chiral symmetry measure $(O)$ and the average thermal Wilson loop $(\bullet)$, calculated on an $8^{3} \times 3$ lattice in the fourth-order hopping parameter expansion. 


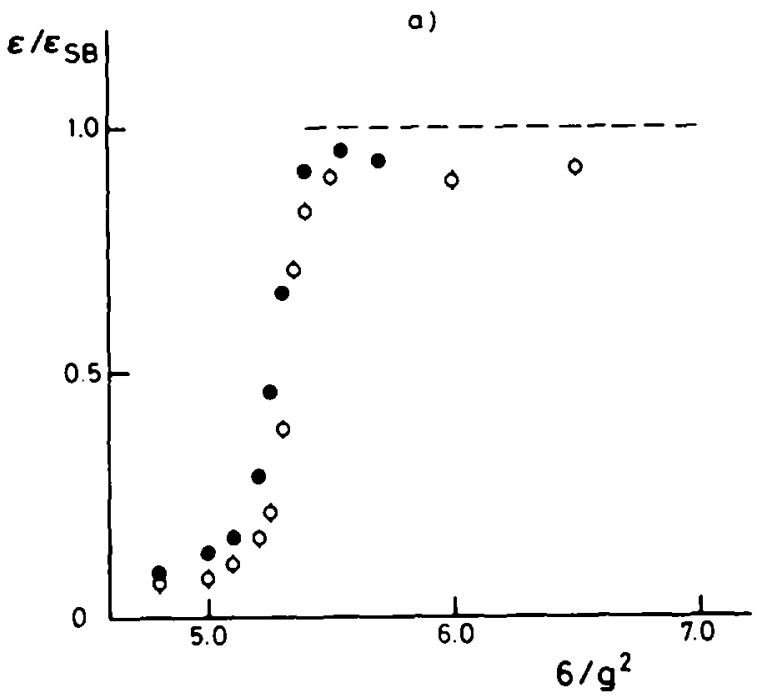

b)

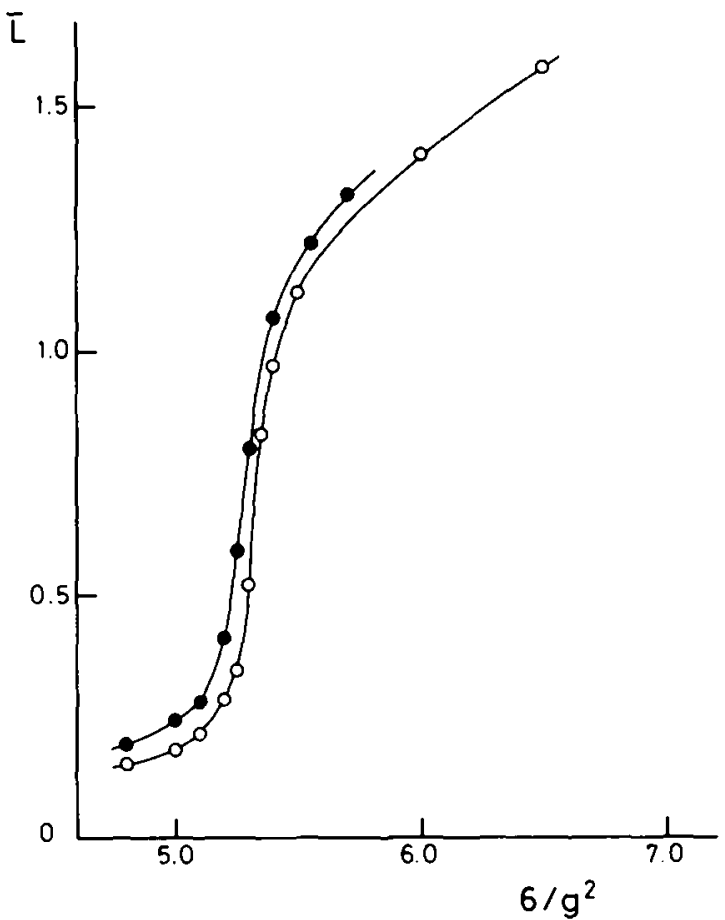

Fig. 8. Comparison of results in the fourth $(O)$ and fifth $(O)$ order hopping parameter expansion on an $8^{3} \times 3$ lattice, for the overall energy density $\varepsilon / \varepsilon_{\mathrm{SB}}(\mathrm{a})$ and for the average thermal Wilson loop (b). 
In fig. 9 , we then show the behaviour of $\varepsilon_{\mathrm{G}}$, as calculated on lattices of size $8^{3} \times 3$, $8^{3} \times 4$ and $10^{3} \times 5$, and normalized in each case to the corresponding StefanBoltzmann form. In fig. 10, the results for $\bar{L}$ on the same set of lattices are presented. The shift in the critical coupling with increasing $N_{\beta}$ is clear; the values of $6 / g_{c}^{2}$, together with the resulting critical temperatures, are listed in table 1 . Within our error margins, we thus do not find any notable deviation from scaling.

The rise of $\varepsilon_{G}$ above the Stefan-Boltzmann limit, which we had already noted before, is now seen to decrease with growing $N_{\beta}$. The technical reason for the overshoot thus becomes evident: the presence of $\operatorname{Re} \bar{L}$ in the effective action causes a more complete ordering on temporal than on spatial links. As a result, $\overline{\mathrm{P}}_{\beta}$ is reduced in comparison to the quenched case, $\overline{\mathrm{P}}_{\mathrm{o}}$ not so much. With increasing $6 / \mathrm{g}^{2}$, all links acquire ordered $U$, and $\varepsilon_{\mathrm{G}}$ returns to its Stefan-Boltzmann limit. When $N_{\beta}$ is increased, the effect of $\operatorname{Re} \bar{L}$ in the effective action is doubly reduced: the factor

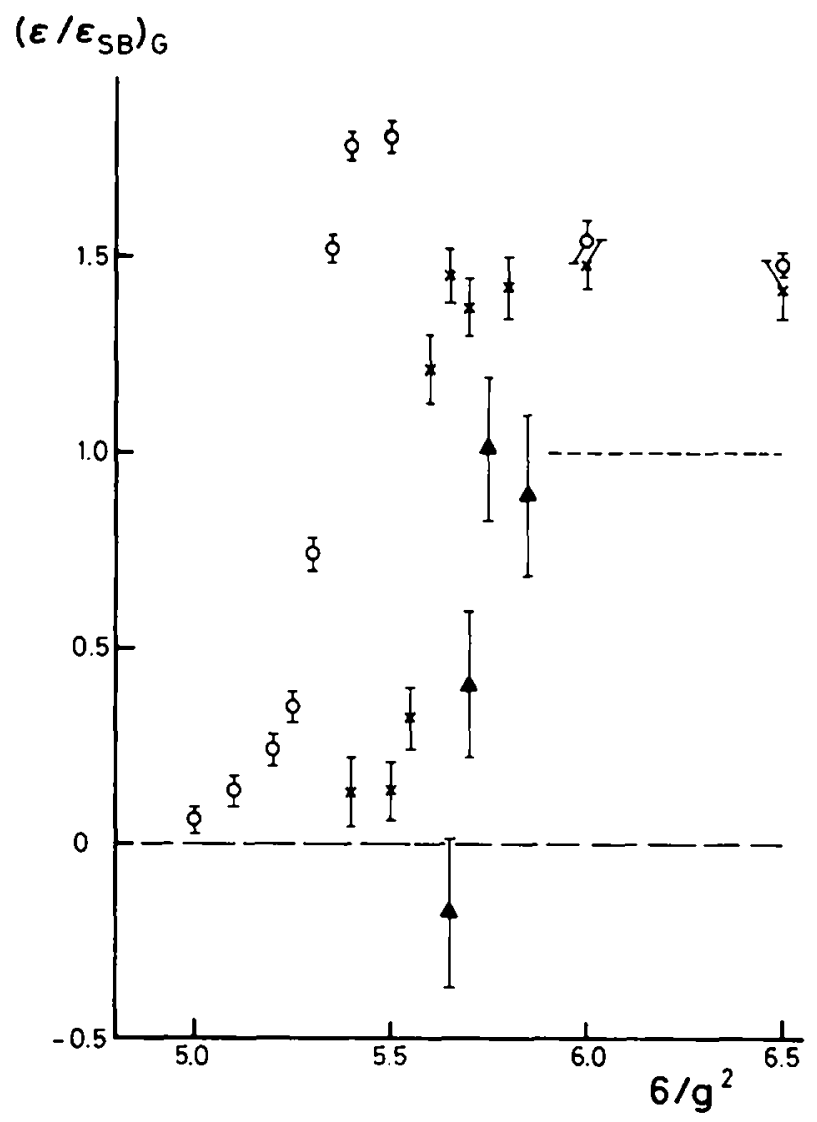

Fig. 9. Comparison of the gluon sector energy density values calculated on an $8^{3} \times 3(0)$, an $8^{3} \times 4(\times)$ and a $10^{3} \times 5(\Delta)$ lattice, normalized in each case to the ideal gas result on a lattice of the same size. 


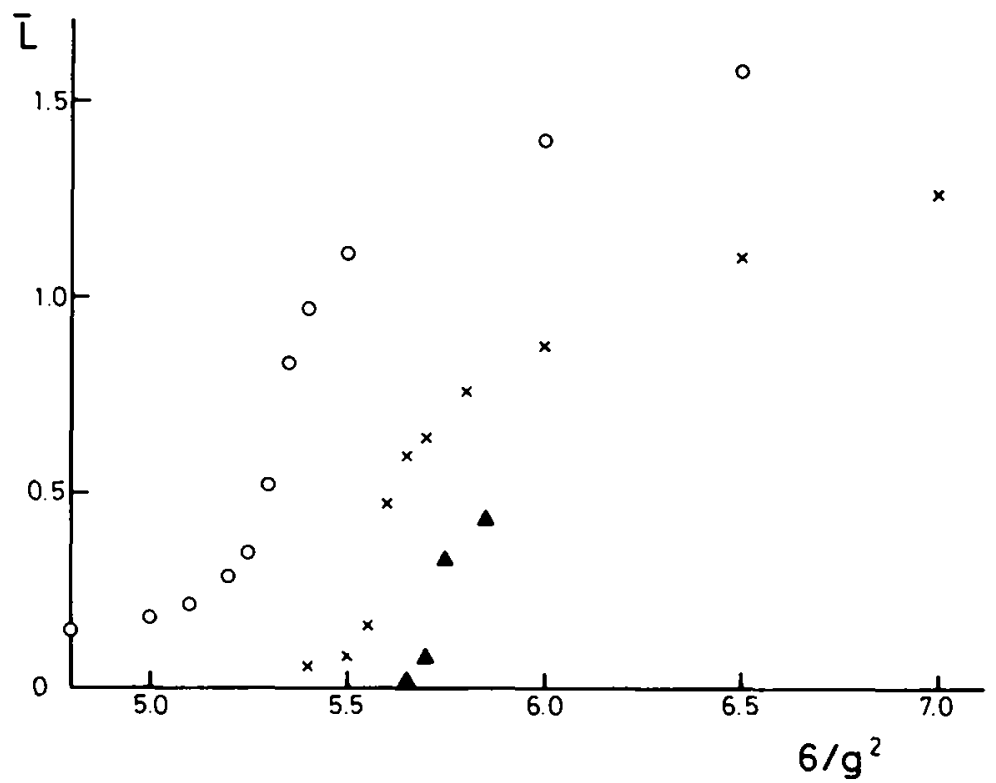

Fig. 10. Comparison of the average thermal Wilson loop values on $8^{3} \times 3(O), 8^{3} \times 4(\times)$ and $10^{3} \times 5$ (A) lattices.

$\kappa^{N_{\beta}}$ becomes smaller, and - as seen from fig. 10 - the value of $\bar{L}$ in the deconfinement zone decreases. This decrease of $\bar{L}$ with $N_{\beta}$ is known from pure gauge theory [23]; it is due to the fact that asymptotically divergent point-source contributions for the colour charge still have to be removed to obtain a physically meaningful observable. In pure gauge theory, this is possible in the weak coupling limit [21]. Here, with a finite number of terms in the hopping parameter expansion, it is not clear how this could be carried out in the action. Hence, in the transition region the energy densities calculated at different $N_{\beta}$ will in general not coincide as functions of $T$. It would be interesting to see if other fermion schemes can avoid this problem, or if it is a general normalization difficulty for the quark-quark interaction on the lattice.

Having seen that the critical temperature is rather insensitive to the temporal lattice size, let us return to its dependence on the order of the hopping parameter expansion. We had already observed that higher order implied smaller $6 / g_{\mathrm{c}}^{2}$. In

TABl.E 1

Critical couplings and temperatures

\begin{tabular}{ccl}
\hline$N_{\beta}$ & $6 / 8_{c}^{2}$ & $T_{c} / A_{L^{\prime}}^{N^{\prime}=2}$ \\
\hline 3 & $5.300 \pm 0.050$ & $152 \pm 10$ \\
4 & $5.575 \pm 0.025$ & $162 \pm 5$ \\
5 & $5.725 \pm 0.025$ & $157 \pm 5$ \\
\hline
\end{tabular}


TABI.E 2

Critical parameters in different fermion schemes

\begin{tabular}{lccccc}
\hline \multicolumn{1}{c}{ Scheme } & $N_{\beta}$ & $N_{f}$ & $6 / g_{c}^{2}$ & $T_{c} / A_{t .}^{0}$ & Ref. \\
\hline Wilson $\kappa^{4}$ & 3 & 2 & $5.30 \pm 0.05$ & $131 \pm 8$ \\
Wilson $\kappa^{5}$ & 3 & 2 & $5.25 \pm 0.05$ & $123 \pm 8$ & this paper \\
KS, canonical & 4 & 3 & 5.3 & 100 & 11 \\
KS, canonical & 2 & 2 & 4.6 & 89 & 12 \\
KS, micro-canonical & 4 & 4 & 5.1 & 106 & 13 \\
\hline
\end{tabular}

table 2 we list our results on the $8^{3} \times 3$ lattice for fourth and fifth order of the hopping parameter expansion, together with the results from other fermion schemes. To allow a comparison, we convert all critical couplings to temperature values $T_{\mathrm{c}} / \Lambda_{L}^{0}$, where $A_{l}^{0}$ is the lattice scale for $N_{f}=0$; its relation to the $A_{L}^{N^{\prime}}$ with $N_{f} \neq 0$ is known perturbatively [23], and we have used this in the conversion. It is seen that our values tend to lie about $20 \%$ above the average value obtained in other schemes. This gives us some indication of the effect of the truncation in $\kappa^{\prime}$. It must be kept in mind, however, that the perturbative relations between $\Lambda_{L}^{N^{\prime}}$ and $\Lambda_{L}^{0}$ are at present coupling values very likely not reliable; hence we do not believe that the $T_{\mathrm{c}} / \Lambda_{L}^{0}$. values in table 2 can be used to obtain $T_{\mathrm{c}}$ in physical units by using pure gauge theory values for $A_{L}^{0}$.

Finally, we want to return to the size of the deconfinement measure $\bar{L}$ in the region below $T_{\mathrm{c}}$. As we have seen in figs. 4 and $10, \bar{L}$ is indeed very small there, so that the $Z_{3}$ symmetry appears to be only weakly broken. To test this in more detail, we have studied the distribution of $L(x)$ values over the lattice in different equilibrium configurations; the results on an $8^{3} \times 4$ lattice are shown in fig. 11. We see in fig. 11a that in the deconfined state, the symmetry is clearly broken, while in the confinement regime, as shown in fig. $11 \mathrm{~b}$, the symmetry is only slightly perturbed. Note that the lattice average of $|L(x)|$ is practically the same in the two cases: it is really the near-restoration of $Z_{3}$ symmetry which leads to the small $\bar{L}$ value below $T_{\text {c }}$.

\section{Conclusions}

We thus find that in the presence of dynamical quarks deconfinement persists as a transition phenomenon between two distinct regimes - and this is as clearly evident here as it is in pure gauge theory. The transition brings the system from a temperature region in which the global $Z_{3}$ symmetry is almost unbroken to one where it is completely absent. At the same point, chiral symmetry is essentially restored. In the Wilson scheme, the functional connection between deconfinement and chiral symmetry restoration is particularly transparent; as can be seen in eq. (18), it is the rapid change of $\bar{L}$ which drives the chiral symmetry restoration in addition to signalling the onset of deconfinement. 


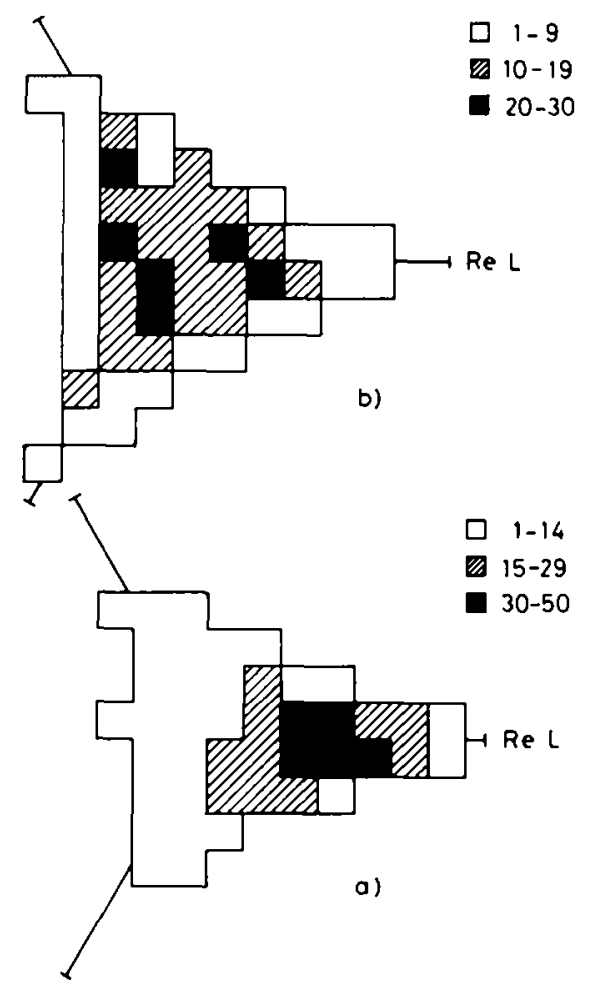

Fig. 11. The distribution of thermal Wilson loop values in the complex $L$ plane for one equilibrium configuration on an $8^{3} \times 4$ lattice. Part (a) is above $T_{c}$, at $6 / g^{2}=6.0$, where $|\bar{L}|=1.08$; part (b) is below $T_{c}$, at $6 / g^{2}=5.4$, with $|\bar{L}|=0.89$.

The change in energy density we observe at deconfinement agrees well with that expected when going from an ideal meson gas to an ideal chromoplasma. The change occurs over an interval of $30 \Lambda_{L^{\prime}=2}^{N^{\prime}=2}$ or less, i.e. for something like a $45 \mathrm{MeV}$ increase in temperature ${ }^{\star}$. All our results indicate a continuous transition - but in view of the truncated hopping parameter expansion we cannot really exclude a first-order transition.

Neither $\bar{L}$ nor $\Delta\langle\bar{\psi} \psi\rangle$ represent genuine order parameters in our formulation; $\bar{L}$ because of the $Z_{3}$ symmetry breaking by dynamical quarks, $\Delta\langle\bar{\psi} \psi\rangle$ because we are not calculating at zero quark mass. These quantities should therefore provide us with some information about the physics underlying the respective symmetry breaking. In the chirally symmetric state, $\Delta\langle\bar{\psi} \psi\rangle$ should give some indication of the effective quark mass at the coupling in question. It is not clear to us how this can be extracted from our data or from that obtained in other fermion schemes. To

- Here, as in figs. 5 and 12 , we have to relate $A_{1^{\prime}}^{N^{-2}}$ to physical units. For illustration purposes, we have taken $A_{L^{\prime}}^{N^{\prime}=2}=1.5 \mathrm{MeV}$. 


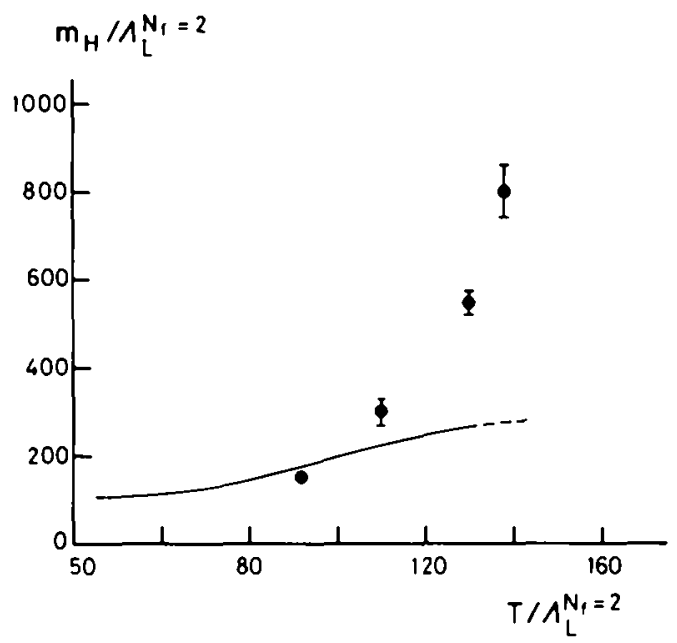

Fig. 12. Values of the effective hadron mass $m_{H}$ obtained from the average thermal Wilson loop, compared to the average hadron mass in an ideal resonance gas with $\pi, p$ and $\omega$ (solid line).

obtain some idea of the quark mass values corresponding to our values of $\kappa\left(g^{2}\right)$, we therefore consider the approximation [25]

$$
\frac{1}{2}\left(\frac{1}{\kappa}-\frac{1}{\kappa_{\mathrm{c}}}\right)=\exp \left(m_{\mathrm{q}} a\right)-1,
$$

where $\kappa_{\mathrm{c}}$ is the (numerical) $\kappa$-value obtained at the point where the pion mass vanishes. Using the results of refs. $[25,26]$ as shown in fig. 1 , we obtain $m_{\mathrm{q}} a \simeq 0.31$ and thus $m_{\mathrm{q}} / T_{\mathrm{c}} \approx 0.94$.

As indicated above, $\bar{L}$ in the confined regime should tell us something about the mass of the dominant hadron state formed when we try to break a string. In fig. 12, we show the mass values $m_{\mathrm{H}}$ obtained from our $\bar{L}$ data using eq. (2), together with the average mass in a meson gas of the $\pi, \rho$ and $\omega$. According to dual models [27] or statistical bootstrap arguments [28], $\bar{m}_{\mathrm{H}}$ should increase as we approach $T_{\mathrm{c}}$. Fig. 12 is seen to be at least qualitatively in accord with our interpretation of $\bar{L}$ in the confinement region.

It is a pleasure to thank R.V. Gavai, P. Hasenfratz, F. Karsch, J. Polónyi and A. Ukawa for stimulating discussions, and the Bochum Computer Center for providing us with the facility (CYBER 205) and computer time.

\section{References}

[1] A.M. Polyakov, Phys. Lett. 72B (1978) 477;

L. Susskind, Phys. Rev. D20 (1979) 2610;

C. Borgs and E. Seiler, Nucl. Phys. B215 (1983) 125 
[2] H. Satz, Nucl. Phys. A418 (1984) 447c

[3] N.F. Mott, Rev. Mod. Phys. 40 (1968) 677 and references given there

[4] T. Çelik, J. Engels and H. Satz, Phys. Lett. 125B (1983) 411;

J. Kogut, M. Stone, H.W. Wyld, W.R. Gibbs, J. Shigemitsu, S.H. Shenker and D.K. Sinclair, Phys. Rev. Lett. 50 (1983) 353

[5] V. Alessandrini, Orsay preprint LPTHE 84/14 (1984)

[6] T. Banks and A. Ukawa, Nucl. Phys. B225 [FS9] (1983) 145

[7] C. De Tar and T. De Grand, Nucl. Phys. B225 [FS9] (1983) 590

[8] P. Hasenfratz, F. Karsch and I.O. Stamatescu, Phys. Lett. 133B (1983) 221

[9] F. Green and F. Karsch, Nucl. Phys. B238 (1984) 297

[10] T. Celik, J. Engels and H. Satz, Phys. Lett. 133B (1984) 427

[11] F. Fucito and S. Solomon, Cal. Tech. preprint CALT-68-1084 (1984):

F. Fucito, C. Rebbi and S. Solomon, Cal. Tech. preprint CALT-68-1124; Nucl. Phys. B248 (1984) 615

[12] R.V. Gavai, M. Lev and B. Petersson, Bielefeld preprint BI-TP 84/10 (1984)

[13] J. Polónyi, H.W. Wyld, J. B. Kogut, J. Shigemitsu and D.K. Sinclair, Phys. Rev. Lett. 53 (1984) 644

[14] K. Wilson, Phys. Rev. D10 (1974) 245; in New phenomena in subnuclear physics, ed. A. Zichichi (Plenum, New York, 1977) Erice, 1975

[15] J. Engels, F. Karsch, I. Montvay and H. Satz, Nucl. Phys. B205 [FS5] (1982) 545

[16] C.B. Lang and H. Nicolai, Nucl. Phys. B200 [FS4] (1982) 135;

A. Hasenfratz and P. Hasenfratz, Phys. Lett. 104B (Sept. 1981) 489

[17] N. Kawamoto, Nucl. Phys. B190 [FS3] (1981) 617

[18] H. Satz, in Quark Matter '84, ed. K. Kajantie (Springer, Berlin, 1985)

[19] R.C. Trinchero, Nucl. Phys. B227 (1983) 61

[20] J. Engels, F. Karsch and H. Satz, Nucl. Phys. B205 [FS5] (1982) 239

[21] U. Heller and F. Karsch, Nucl. Phys. B251 [FS13] (1985) 254

[22] T. Çelik, J. Engels and H. Satz, Phys. Lett. 129B (1983) 323

[23] W. Celmaster and D.J. Maloof, Phys. Rev. D24 (1981) 2730;

H. Kawai, R. Nakayama and K. Seo, Nucl. Phys. B189 (1981) 40

[24] R.V. Gavai, F. Karsch and H. Satz, Nucl. Phys. B220 [FS8] (1983) 223

[25] A. Hasenfratz, Z. Kunszt, P. Hasenfratz and C.B. Lang, Phys. Lett. 110B (1982) 289

[26] H. Lipps, G. Martinelli, R. Petronzio and F. Rapuano, Phys. Lett. 126B (1983) 250;

P. Hasenfratz and I. Montvay, DESY preprint 87-072 (1983)

[27] M. Jacob (ed.), Dual theory (North-Holland, Amsterdam, 1974)

[28] R. Hagedorn, CERN Report 71-12 (Yellow Series, 1971) 